\title{
Lógica minimalista: símbolos, linguagens, termos e fórmulas
}

\author{
Colaboração Matemática Aberta ${ }^{1}$ \\ 10 de Fevereiro de 2022
}

\begin{abstract}
Resumo
Este é o primeiro artigo da série "Lógica minimalista". Apresentamos alguns conceitos fundamentais da lógica clássica, são eles, os símbolos, as linguagens, os termos e as fórmulas.
\end{abstract}

palavras-chave: fundamentos da lógica clássica, linguagem, termo, fórmula

A versão mais atualizada deste artigo está disponível em

https://osf.io/qjmch/download

https: //zenodo.org/record/5383409

\section{Preâmbulo}

1. Mathematics is the Queen of the Sciences (Gauss).

2. Logic is the Queen of Mathematics.

3. Este white paper foi adaptado de [1], seguindo a licença Creative Commons [2].

4. No final deste artigo, você terá aprendido o que é uma linguagem formal na matemática.

${ }^{1}$ Todos os autores com suas afiliações aparecem no final deste artigo. 


\section{Pré-requisitos}

5. Recomendamos fortemente consultar este excelente livro [3], caso você se depare com algum conceito que ainda não saiba.

\section{Introdução}

6. A lógica faz o link entre matemática e filosofia.

7. A lógica faz, também, o link entre matemática e ciência da computação teórica.

\section{Símbolos metalinguísticos}

8. Utilizaremos dois símbolos metalinguísticos.

9. O símbolo := indica que o que aparece à esquerda é definido como o que aparece à direita.

10. O símbolo :三 está definido mais adiante [veja (56)], pois precisamos, primeiro, de alguns pré-requisitos.

\section{Axiomas}

11. axioma $:=$ verdade matemática evidente que não precisa ser demonstrada

12. sistema de axiomas := conjunto de axiomas

\section{Modelos ou estruturas matemáticas}

13. Modelo é sinônimo de estrutura matemática. 
14. A seguir estão dois exemplos de estruturas matemáticas, apresentadas com o intuito de informar ao leitor o jargão matemático.

15. Um grupo é um modelo (ou estrutura matemática) de um conjunto de axiomas.

16. $\mathbb{R}^{3}$, que é uma coleção de trios ordenados de números reais, é um modelo (ou estrutura matemática) de axiomas do espaço vetorial.

\section{LINGUAGENS}

17. A sintaxe de uma linguagem são os símbolos utilizados.

18. sintaxe $:=$ símbolos (de uma linguagem)

19. cadeia de símbolos := sequência de símbolos

20. A partir de agora, a palavra cadeia significa cadeia de símbolos.

\section{Primeira ordem}

21. Definição:

linguagem de primeira ordem $:=$ coleção infinita de símbolos distintos (tal que nenhum esteja contido no outro)

22. Temos oito categorias de símbolos, apresentadas a seguir (a vírgula separa os respectivos símbolos).

23. Parêntesis: $($,$) .$

24. Conectivos: $\vee, \neg$

25. Quantificador: $\forall$.

26. Variáveis (uma para cada inteiro positivo $n$ ): $v_{1}, v_{2}, \ldots, v_{n}, \ldots$ Vars $:=$ conjunto de símbolos de variáveis 
27. Igualdade: $=$.

28. Constante: para cada $n$ inteiro positivo, algum conjunto contendo zero ou mais símbolos.

29. Função: para cada $n$ inteiro positivo, algum conjunto contendo zero ou mais símbolos de funções $n$-árias.

30. Relação: para cada $n$ inteiro positivo, algum conjunto contendo zero ou mais símbolos de relações $n$-árias.

\section{Comentário sobre aridade}

31. O símbolo para uma função $n$-ária significa que ele representa uma função de $n$ variáveis.

32. Por exemplo, o símbolo + tem aridade 2, ou seja, é uma função de duas variáveis.

33. O simbolo para uma relação $n$-ária significa que ele representa uma relação em $n$-uplas de objetos.

\section{Como especificar uma linguagem?}

34. especificar uma linguagem $:=$ determinar quais (se houver) símbolos (constante, função, relação) desejamos utilizar

35. Resumindo, os simbolos constante, função e relação especificam uma linguagem.

36. Note que as variáveis não aparecem na especificação de uma linguagem.

37. Assumiremos que cada linguagem terá o símbolo da igualdade, a menos que seja especificamente indicado. 
38. Especificar uma linguagem consiste em listar os símbolos (constante, função, relação).

39. Curiosamente, existe um infinito não contável de cada um desses símbolos! (veja o apêndice de [1])

\section{Exemplos de linguagens}

40. Um grupo consiste de um conjunto e uma operação binária com certas propriedades, como identidade e inversa.

41. Existe uma certa arbitrariedade na escolha do delineamento de uma linguagem.

42. Considere que $\mathcal{L}_{G}$ represente uma linguagem para grupos.

43. Uma opção é $\mathcal{L}_{G}$ ser $\{0,+\}$, sendo 0 o símbolo constante e + o símbolo da função binária.

44. Outra opção é $\mathcal{L}_{G}$ ser $\{1,-1, \cdot\}$, sendo 1 o símbolo constante, $\cdot$ o simbolo da função binária $\mathrm{e}^{-1} \mathrm{o}$ símbolo da função unária (1-ária), que retorna a inversa de um elemento do grupo.

45. Seja $\mathcal{L}_{T C}$ a linguagem para a teoria de conjuntos.

46. $\mathcal{L}_{T C}$ é $\{\epsilon\}$.

47. $\in$ é o símbolo para pertence.

48. $x \in y$ significa que o conjunto $x$ é um elemento do conjunto $y$.

49. Outros simbolos, como contido ( $\subset$ ) e $\varnothing$ podem ser definidos a partir de outros mais primitivos. 


\section{Significado}

50. Suponha que $\mathcal{L}$ seja a linguagem $\{0,+,<\}$.

51. Note que a cadeia de símbolos

$$
\left(v_{1}+0\right)<v_{1}
$$

tem significado, ainda que seja falsa.

52. Por outro lado, a cadeia de símbolos

$$
\left.v_{12}\right)(\forall++<(0
$$

não tem significado.

\section{TERMOS}

53. Termos são cadeias de símbolos que se referem aos objetos da linguagem.

54. Definição

termo de $\mathcal{L}:=$ cadeia não vazia e finita $t$ de símbolos de $\mathcal{L}$ que satisfazem exatamente uma das seguintes condições:

(a) t é um simbolo constante, ou

(b) $t$ é uma variável, ou

(c) $t: \equiv f t_{1} t_{2} \ldots t_{n}$, sendo $f$ um símbolo de função $n$-ária de $\mathcal{L}$ e cada um dos $t_{i}$ é um termo de $\mathcal{L}$.

55. Note que uma relação de símbolos (que não seja função) não é um termo.

56. O símbolo metalinguístico :三 significa que as cadeias de $\mathcal{L}$-símbolos de cada lado de : sã̃o idênticas.

57. $\mathcal{L}$-símbolos significa os simbolos da linguagem $\mathcal{L}$. 
58. Note que (54.c) é uma definição por recursão, dado que $t$ é um termo se contiver subcadeias que são termos.

59. Subcadeia é um subconjunto de uma cadeia de símbolos.

\section{Leitura única}

60. Existe uma única maneira de se ler qualquer sequência de símbolos matemáticos para evitar interpretações ambíguas.

\section{FÓRMULAS}

61. Fórmulas são afirmações acerca dos objetos de uma estrutura matemática (modelo).

\section{Notação polonesa}

62. Antes de definirmos o que é uma fórmula, precisamos mostrar como ler uma notação polonesa.

63. Seja $\mathcal{L}$ a linguagem $\{\overline{0}, \overline{1}, \overline{2}, \ldots,+, \cdot\}$ com um símbolo constante para cada número natural e dois símbolos de funções binárias.

64. $+\overline{3} \overline{2}$ significa $(\overline{3}+\overline{2})$, que é igual a $\overline{5}$.

$65 .+\overline{3} \overline{2} \overline{4}: \equiv(\overline{3}+\overline{2}) \cdot \overline{4}: \equiv \overline{5} \cdot \overline{4}: \equiv \overline{20}$

66. De (54), temos que $\overline{123},+\overline{3} \overline{2}$ e $\cdot+\overline{3} \overline{2} \overline{4}$ são termos de $\mathcal{L}$, enquanto que $\overline{1} \overline{2} \overline{3}$ não é um termo de $\mathcal{L}$, mas sim uma sequência de três termos.

67. Em notação polonesa, essas duas cadeias de símbolos são equivalentes, $f t_{1} t_{2} \ldots t_{n}: \equiv f\left(t_{1}, t_{2}, \ldots, t_{n}\right)$. 


\section{Fórmula}

68. Definição

fórmula de $\mathcal{L}: \equiv$ cadeia $\phi$ não vazia e finita de símbolos de $\mathcal{L}$ que satisfazem exatamente uma das seguintes condições:
(a) $\phi: \equiv=t_{1} t_{2}$, sendo $t_{1}$ e $t_{2}$ termos de $\mathcal{L}$, ou
(b) $\phi: \equiv R t_{1} t_{2} \ldots t_{n}$, sendo $R$ um símbolo de uma relação $n$-ária de $\mathcal{L}$, e $t_{1}, t_{2}, \ldots, t_{n}$ são termos de $\mathcal{L}$, ou
(c) $\phi: \equiv(\neg \alpha)$, sendo $\alpha$ uma fórmula de $\mathcal{L}$, ou
(d) $\phi: \equiv(\alpha \vee \beta)$, sendo $\alpha$ e $\beta$ fórmulas de $\mathcal{L}$, ou
(e) $\phi: \equiv(\forall v)(\alpha)$, sendo $v$ uma variável e $\alpha$ uma fórmula de $\mathcal{L}$.

69. Note que (68.c,d,e) são definições por recursão, dado que $\phi$ é uma fórmula se contiver outras fórmulas.

70. Em (68.e), dizemos que o alcance do quantificador $\forall$ é $\alpha$.

\section{Fórmulas atômicas}

71. As fórmulas atômicas de $\mathcal{L}$ são aquelas que satisfazem a condição (a) ou (b) de (68),
(a) $\phi: \equiv=t_{1} t_{2}$,
(b) $\phi: \equiv R t_{1} t_{2} \ldots t_{n}$.

72. Essas fórmulas são denominadas atômicas porque são as primitivas, já que as outras três condições de (68) são recursivas, isto é, definem uma fórmula a partir de outra fórmula.

73. Veja mais sobre recursão em [4]. 


\section{Qual a diferença entre termos e fórmulas?}

74. Um termo não é uma fórmula!

75. Fazendo uma comparação com o idioma português, por exemplo, o termo é análogo a uma palavra e uma fórmula é análoga a uma afir$\operatorname{mação.~}$

76. Uma fórmula pode ser verdadeira ou falsa.

77. $f t_{1} t_{2} \ldots t_{n}$ é um termo.

78. $R t_{1} t_{2} \ldots t_{n}$ é uma fórmula.

\section{Considerações Finais}

79. No item a seguir, apresentamos uma lista de palavras-chave que constituem conceitos fundamentais que devem ser compreendidos e lembrados, para que seus estudos em lógica ocorram com maior fluidez.

80. símbolos, simbolos metalinguísticos, axioma, sistema de axiomas, modelo, estrutura matemática, sintaxe, cadeia de símbolos, linguagem de primeira ordem, parêntesis, conectivos, quantificador, variáveis, igualdade, constante, função n-ária, relação n-ária, especificar uma linguagem, significado, termos, recursão, subcadeia, fórmulas, notação polonesa, alcance do quantificador, fórmulas atômicas

81. Se você compreendeu bem cada um desses conceitos-chaves, está pronta(o) para estudar o próximo artigo desta série! [4]

82. Para finalizar, considere a seguinte regra mnemônica (em um diagrama não formal) para ajudar na memorização dos conceitos apresentados: 


$$
\begin{gathered}
\mathcal{L}[c f R], \\
t[c v f], \\
\text { formula }[=, R, \neg, \vee, \forall v] .
\end{gathered}
$$

\section{Ciência Aberta}

O arquivo latex para este artigo, juntamente com outros arquivos suplementares, estão disponíveis em [5]. Seja coautor(a) deste artigo, envie sua contribuição para mplobo@uft. edu.br.

\section{Consentimento}

O autor concorda com [6].

\section{Como citar este artigo?}

https://doi.org/10.31219/osf .io/qjmch

https://zenodo.org/record/5383409

\section{Licença}

CC-By Attribution 4.0 International [2]

\section{Referências}

[1] Leary, Christopher C., and Lars Kristiansen. A friendly introduction to mathematical logic, 2nd edition, 2015.

https://knightscholar geneseo . edu/geneseo-authors/6/ 
[2] Creative Commons. Attribution-NonCommercial-ShareAlike 4.0 International (CC BY-NC-SA 4.0).

https://creativecommons.org/licenses/by-nc-sa/4.0

[3] Velleman, Daniel J. How to prove it: A structured approach. Cambridge University Press, 2019.

[4] Lobo, Matheus P. "Lógica Minimalista: Indução." OSF Preprints, 13 Oct. 2020. https://doi.org/10.31219/osf.io/j4fdx

[5] Lobo, Matheus P. "Open Journal of Mathematics and Physics (OJMP)." OSF, 21 Apr. 2020.

https://doi.org/10.17605/osf .io/6hzyp

[6] Lobo, Matheus P. "Simple Guidelines for Authors: Open Journal of Mathematics and Physics." OSF Preprints, 15 Nov. 2019.

https://doi.org/10.31219/osf .io/fk836

\section{Colaboração Matemática Aberta}

Matheus Pereira Lobo ${ }^{1,2,3}$ (autor principal, mplobo@uft.edu.br) https://orcid.org/0000-0003-4554-1372

${ }^{1}$ Universidade Federal do Tocantins (Brasil)

${ }^{2}$ Universidade Federal do Norte do Tocantins (Brasil)

${ }^{3}$ Universidade Aberta (UAb, Portugal) 